\title{
O Efeito Miragem no Estudo de Processos Electroquímicos
}

\author{
L. M. ABRANTES * E M. C. OLIVEIRA *
}

\section{O Efeito Miragem constitui uma técnica óptica in-situ de monitorização do gradiente de indice de refracção de uma solução, o qual pode relacionar-se directamente com o gradiente de concentração da interface eléctrodo-electrólito. O crescente interesse pela técnica justifica-se pela possibilidade de seguir os fluxos iónicos na vizinhança da superfície do eléctrodo, permitindo assim elucidar mecanismos reaccionais de processos electródicos. \\ Para uma melhor compreensào do contributo do Efeito Miragem na investigação de sistemas electroquímicos, focam-se os aspectos teóricos fundamentais, apresentam-se alguns exemplos ilustrativos e discutem-se as perspectivas de futuros desenvolvimentos.}

\section{INTRODUÇÃO}

As miragens, fenómenos que ocorrem na natureza, particularmente nos desertos ou em regiões árcticas, são explicáveis pelo efeito da deflexão da luz solar em meios de índice de refracção variável, resultante de variações de temperatura do ar na vizinhança do solo. Por analogia, designou-se por Efeito Miragem a técnica introduzida por Boccara e col. em 1980 [1], baseada na medição do desvio de um feixe laser atravessando um meio transparente de índice de refracção variável.

A variação do índice de refracção pode ser induzida por um gradiente de temperatura (Efeito Miragem de Temperatura, ou Espectroscopia Deflexional Fototérmica,) e/ou por um gradiente de concentração (Efeito Miragem de Concentração, ou Deflexão do Feixe Laser de acordo com a terminologia inglesa, Probe Beam Deflection). Contrariamente à vasta aplicação da técnica em estudos fototérmicos [2], a utilização em que o feixe laser funciona como sensor do gradiente de concentração tem sido pouco explorada. No entanto, esta técnica pode constituir um importante utensílio, não apenas no estudo de sistemas químicos, mas também físicos e biológicos, i.e., sempre que se deseje seguir a formação de um gradiente de concentração numa interface heterogénea.

Neste trabalho pretende-se ilustrar o importante contributo do Efeito Miragem de Concentração na investigação de sistemas electroquímicos.

Quando um condutor electrónico em contacto com uma solução electrolítica é sujeito a um potencial, ou a uma cơrrente eléctrica, origina um fluxo iónico na camada de difusão, que resulta na formação de um gradiente de concentração. Este gradiente pode ser detectado por um feixe laser, alinhado paralelamente e o mais próximo possível do eléctrodo, que ao atravessar o meio de índice de refracção variável, se desvia no sentido da maior concentração das espécies, ou seja, do maior índice de refracção, figura 1.

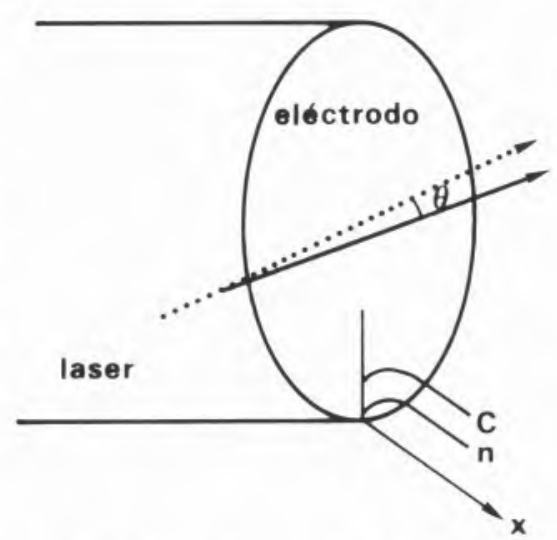

Fig. 1 - Esquema da variação de trajectória de um feixe ao atravessar um meio de índice de refracção variável. $O$ feixe é deflectido na direcção do maior índice de refracção.

Se o sentido e magnitude da deflexão do feixe permitem determinar, respectivamente, a direcção predominante e a extensão do fluxo iónico, o registo simultâneo da corrente eléctrica possibilita inferir a carga associada às espécies iónicas. Por exemplo, no caso de se registar uma carga anódica concomitante com o registo de um valor negativo de $\theta(x, t)$ (por convenção corresponde a um desvio do feixe aproximando-se do eléctrodo, ou seja, um fluxo de matéria, $J(x, t)$, do eléctrodo para o seio da solução), poder-se-á concluir que a reacção de oxidação envolve a formação de espécies de carga positiva $\left(\mathrm{C}^{+}\right)$. O facto do mesmo processo electródico poder ser acompanhado por um $\theta(x, t)$ positivo (corresponde a um fluxo de matéria do seio da solução para o eléctrodo) permite diagnosticar que a reacção de oxidação envolve o consumo de espécies iónicas, de carga negativa, $\left(\mathrm{A}^{-}\right)$, figura 2.

Assim, o acoplamento do Efeito Miragem a técnicas electroquímicas correntes (por exemplo, voltametria cíclica e cronoamperometria) apresenta-se como uma metodologia simples, não destrutiva e de elevada sensibilidade, para estudar in-situ processos electroquímicos [3]. Usualmente a deflexão angular situa-se no intervalo $40^{-3}$ a $10^{-6}$ radiano, sendo o limite de detecção de cerca de I nrad. A elevada sensibilidade da técnica foi ilustrada no estudo da dissolução/deposição de cobre tendo sido possível detectar a formação de 0.02 de uma monocamada daquele metal [4].

\section{Relação entre o desvio angular} e o gradiente de concentração

À pressão e temperatura constantes, o desvio de um feixe laser, ao atravessar um meio opticamente não homogéneo, pode ser descrito pela seguinte expressão,

$$
\theta_{0}(x, t)=\frac{L}{n_{0}}\left(\frac{\partial n}{\partial c}\right) \cdot\left(\frac{\partial c}{\partial x}\right)
$$

na qual $\theta_{0}(\mathrm{x}, \mathrm{t})$ representa o desvio do feixe, no interior da célula electroquímica, a uma distância $\mathrm{x}$ do eléctrodo no tempo t, L o compri-mento do eléctrodo, $\frac{\partial \mathrm{c}}{\partial \mathrm{x}}$ o gradiente de concentração, $\frac{\partial n}{\partial c}$ a variação do índice de refracção com a variação da concentração e $\mathrm{n}_{\mathrm{o}} \mathrm{o}$ índice de refracção médio no seio da solução electrolítica. Nesta expressão são tidas em consideração algumas simplifica- 

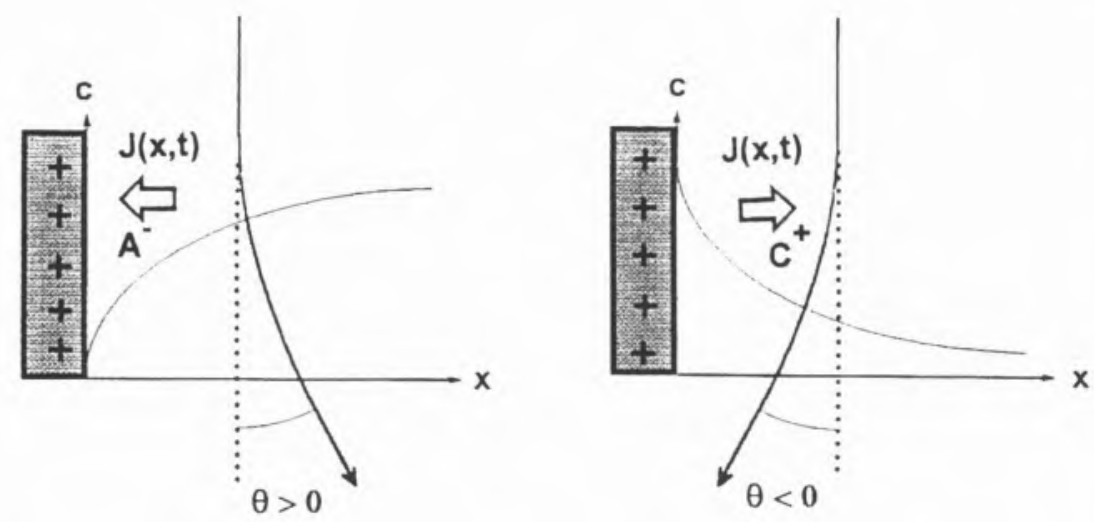

Fig. 2 - Esquema da relação entre o fluxo iónico $\mathbf{J}(x, t)$ e o desvio do feixe, $\theta$, numa reacção de oxidação.

ções da equação geral que descreve a propagação de um feixe: assume-se que o feixe é infinitamente fino e que o desvio é pequeno, comparativamente à espessura do gradiente de concentração (uma deflexão de 1 mrad corresponde a um afastamento de $5 \mu \mathrm{m}$ de um eléctrodo de $5 \mathrm{~mm}$ de diâmetro; valor significativamente menor que a espessura do gradiente de concentração, tipicamente com valores superiores a $10 \mu \mathrm{m}$ [5]).

Experimentalmente, mede-se o desvio do feixe no exterior da célula electroquímica, $\theta(x, t)$, que se relaciona com $\theta_{0}(x, t)$ pela lei de SnellDescartes $\left(n \operatorname{sen} \theta=n_{0} \operatorname{sen} \theta_{0}\right)$. Assim, admitindo que o índice de refracção do ar, n, é 1, a equação (1) é substituída por,

$$
\theta(x, t)=L\left(\frac{\partial n}{\partial c}\right) \cdot\left(\frac{\partial c}{\partial x}\right)
$$

Em sistemas onde mais que uma espécie contribui para o gradiente de índice de refracção, o desvio do feixe é igual ao somatório da contribuição de cada espécie.

$\theta(x, t)=L \times\left[\sum_{i} \frac{\partial n}{\partial c_{i}} \times \frac{\partial c_{i}(x, t)}{\partial x}\right]$

Fazendo uso desta equação, é possível simular a resposta do feixe laser para um dado mecanismo reaccional e compará-la com os resultados experimentais. Com efeito, uma vez que a aplicação da equação 3 implica calcular o gradiente de concentração de cada uma das espécies partindo de um modelo pré-estabelecido, pode pois testar-se o modelo por comparação dos gradientes de concentração calculados teoricamente com os determinados experimentalmente, ou seja, por comparação da curva de deflexão simulada com a experimental.

A determinação do gradiente de concentração no electrólito tem incontestáveis vantagens face a registos puramente electroquímicos. Enquanto estes estão somente sujeitos à medição da transferência de massa na fronteira, $x=0$, ou mais concretamente à transferência de carga, o gradiente de concentração resultante da medição da deflexão do feixe laser é sensível a pelo menos dois pontos na camada de difusão, a $\mathrm{x}=0$ e $\mathrm{x}=\mathrm{x}_{0}+\mathrm{d}$ (sendo $\mathrm{x}_{0}$ a distância mínima entre o feixe e o eléctrodo, e d a distância ao feixe a que o eléctrodo é posicionado), pelo que também permite detectar a ocorrência de reacções não faradaicas, e.g. processos de adsorção e reacções químicas.

\section{INSTRUMENTAÇÃO}

Os principais componentes da instalação experimental compreendem, para além do equipamento electroquímico (potenciostato e gerador de ondas), a fonte monocromática de feixe laser, o sistema de alinhamento do eléctrodo ao feixe, a célula electroquímica e o sistema da detecção. A figura 3 é ilustrativa do esquema da montagem experimental.

O feixe laser deverá ter essencialmente duas características: uma baixa potência (usualmente $2 \mathrm{~mW}$ ) de modo a não perturbar a estrutura da dupla camada e um pequeno diâmetro que viabilize a sua máxima aproximação à superfície do eléctrodo. Embora dependa da distância focal das lentes utilizadas na convergência do feixe, do diâmetro do feixe antes de focalizado e do eléctrodo, a distância mínima eléctrodo-feixe situa-se, em geral, entre 30-150 $\mu \mathrm{m}$ [6].

A técnica do Efeito Miragem requer que a célula electroquímica tenha duas janelas, paralelas e transparentes, pelas quais o feixe passe perpendicularmente. Vulgarmente, esta célula é constituída por uma "cuvette" de quartzo na qual é inserido paralelamente ou perpendicularmente ao feixe laser, o eléctrodo de trabalho. Contrariamente aos requisitos impostos pela maioria das técnicas de reflectância, poderão ser utilizados eléctrodos de trabalho cujas superfícies sejam rugosas, porosas ou opacas. À semelhança de qualquer célula de três eléctrodos, é igualmente introduzido um eléctrodo de referência e um secundário. A distância entre este último e o eléctrodo de trabalho deverá ser suficientemente grande para que não haja interferência do gradiente de concentração resultante de reaç̧ões que aí ocorram.

O sistema de alinhamento do eléctrodo ao feixe é essencialmente constituído por uma plataforma, na qual o eléctrodo de trabalho é fixo, que permite o movimento motorizado do eléctrodo com uma elevada precisão (tipicamente $0.02 \mu \mathrm{m}$ ), segundo dois graus de liberdade: os movimentos de translação e de rotação. O primeiro permite variar a distância do eléctrodo relativamente ao feixe e, conjuntamente com o segundo, assegurar o paralelismo feixeeléctrodo. 

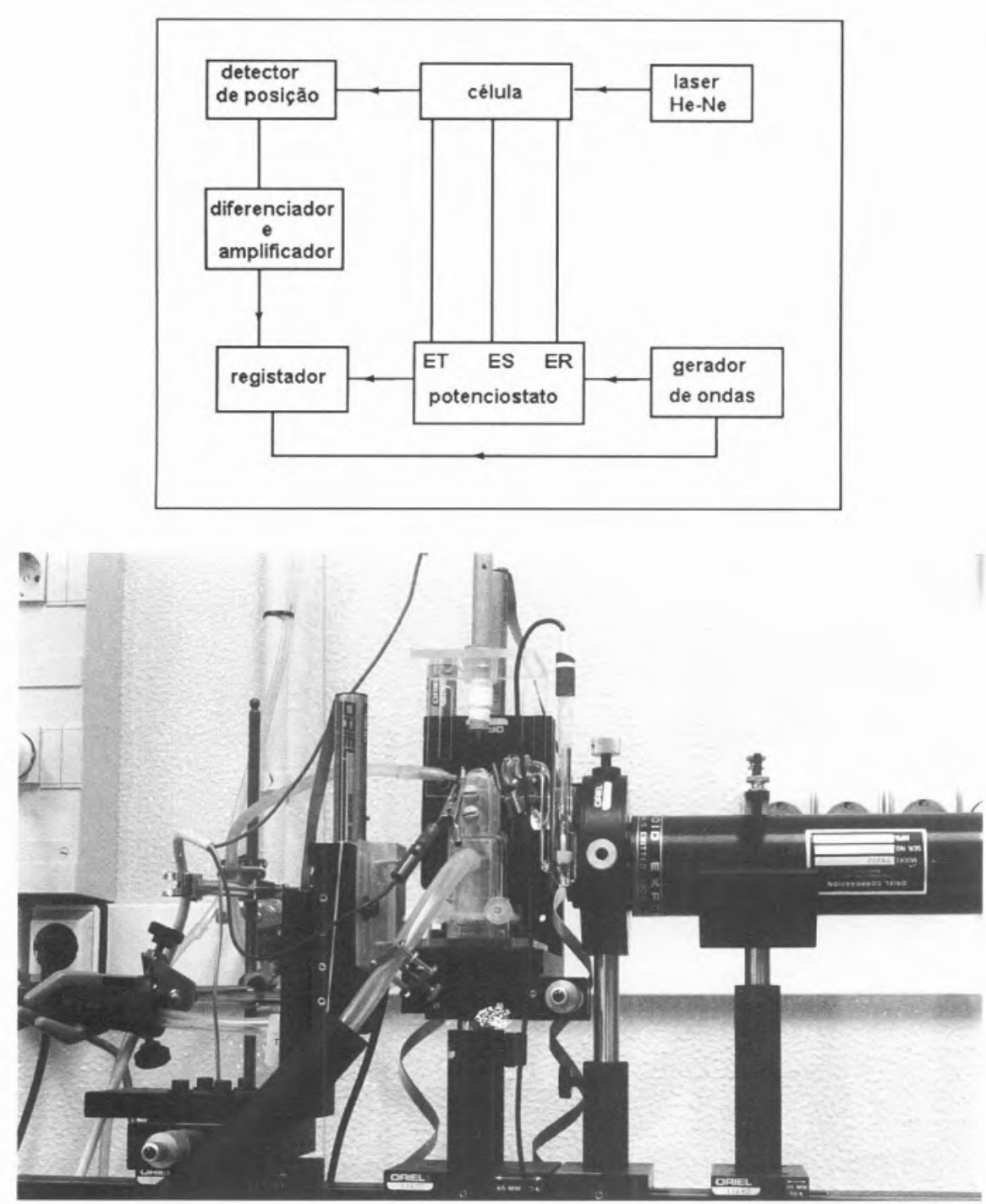

Fig. 3 - Esquema e fotografia da montagem global do Efeito Miragem.

O detector de posição é usualmente um bifotodíodo, com duas superfícies opticamente activas, ligeiramente distanciadas $(\approx 10 \mu \mathrm{m})$, conectado a um circuito electrónico. Dado que o sinal voltaico produzido por cada um dos fotodíodos $\left(\mathrm{V}_{\mathrm{A}}\right.$ e $\left.\mathrm{V}_{\mathrm{B}}\right)$ é dependente da área iluminada pelo feixe laser, o circuito electrónico permitirá medir $\Delta \mathrm{E}=\frac{\left(\mathrm{V}_{\mathrm{A}}-\mathrm{V}_{\mathrm{B}}\right)}{\left(\mathrm{V}_{\mathrm{A}}+\mathrm{V}_{\mathrm{B}}\right)}$ que é proporcional ao desvio do feixe relativamente ao centro do bifotodíodo. Também o bifotodíodo está fixo a uma plataforma motorizada que permite o seu movimento trans-

A aplicação do Efeito Miragem em estudos electroquímicos tem incidido essencialmente na determinação da natureza (catiónica ou aniónica) dos iões que participam no balanço de carga de um filme condutor, por exemplo, polímeros electroactivos ou filmes electrocrómicos sujeito a um processo redox [7-9].
Neste domínio, as aplicações a polímeros modificados encontram-se em franca expansão [10-12]. Registam-se igualmente estudos sobre a deposição/dissolução de filmes metálicos [13,14,] corrosão/passivação de metais $[15,16]$ e electrocatálise $[17,18]$. Apresentam-se alguns exemplos ilustrativos da utilização da técnica.

\section{Estudo de electro-oxidação do cobre}

O estudo da dissolução anódica do cobre é importante na compreensão de mecanismos de corrosão. A oxidação deste metal é bastante complexa, pois dependendo do $\mathrm{pH} \mathrm{e}$ do potencial, poderão formar-se produtos solúveis, tais como Cu(I), $\mathrm{Cu}(\mathrm{II}), \mathrm{Cu}(\mathrm{III}) \mathrm{e} / \mathrm{ou}$ produtos insolúveis, $\mathrm{Cu}_{2} \mathrm{O}, \mathrm{CuO}, \mathrm{Cu}(\mathrm{OH})_{2}, \mathrm{Cu}_{2} \mathrm{O}_{3}$. $\mathrm{O}$ estudo da oxidação do $\mathrm{Cu}$ pelo Efeito Miragem, particularmente em meio alcalino, tem contribuído para esclarecer o mecanismo de formação dos óxidos/hidróxidos [14,19]. A pH 14 [19], por exemplo, embora os estudos voltamétricos revelem a existência de um pico anódico com uma elevada densidade de corrente, a qual é atribuída à formação do $\mathrm{CuO}$.

$\mathrm{Cu}_{2} \mathrm{O}+2 \mathrm{OH}^{-} \rightarrow 2 \mathrm{CuO}+\mathrm{H}_{2} \mathrm{O}+2 \mathrm{e}$

$\mathrm{Cu}+2 \mathrm{OH}^{-} \rightarrow \mathrm{CuO}+\mathrm{H}_{2} \mathrm{O}+2 \mathrm{e}$

os resultados do Efeito Miragem, figura 4, apontam para que este óxido se forme por um outro processo electroquímico. Com efeito, àquele potencial, o feixe deflecte inicialmente no sentido de aproximar do eléctrodo, sendo depois o sinal invertido. Este comportamento indica que existe um aumento inicial da concentração iónica junto ao eléctrodo, o que é consistente com a seguinte reacção,
$\mathrm{Cu} \rightarrow \mathrm{Cu}^{2+}+2 \mathrm{e}$

A posterior deflexão do feixe no sentido de se afastar do eléctrodo revela uma diminuição da concentração iónica junto ao eléctrodo, o que sugere as seguintes reacções: 
$\mathrm{Cu}^{2+}+2 \mathrm{OH}^{-} \rightarrow \mathrm{Cu}(\mathrm{OH})_{2}$

$\mathrm{Cu}(\mathrm{OH})_{2} \leftrightarrow \mathrm{CuO}+\mathrm{H}_{2} \mathrm{O}$

O Efeito Miragem permite pois revelar processos que não são detectáveis por métodos clássicos electroquímicos, contribuindo assim para a elucidação de mecanismos reaccionais.
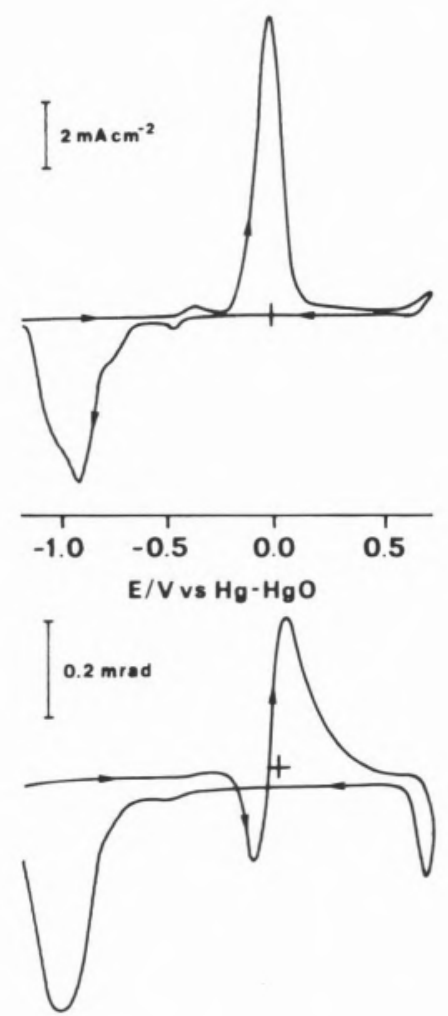

Fig. 4 - Voltamograma cíclico e deflectograma de um eléctrodo de $\mathrm{Cu}$ em $0.1 \mathrm{M} \mathrm{NaOH}^{*}$.

Estudo do mecanismo de conversão redox de polímeros electronicamente condutores (PEC)

O processo electroquímico de oxidação-redução de PEC é acompanhado por troca de iões com a solução electrolítica, para manter a electroneutralidade, figura 5. A concentração iónica em solução altera-se e o gradiente de índice de refracção criado (perpendicular à superfície do eléctrodo) conduz a um desvio do feixe proporcional à extensão e direcção do fluxo iónico.

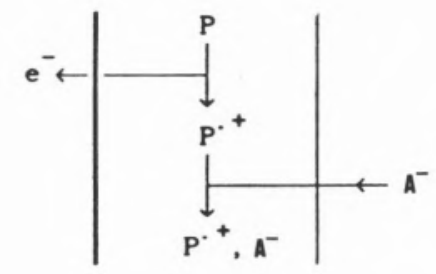

\section{OXIDAÇÃO}

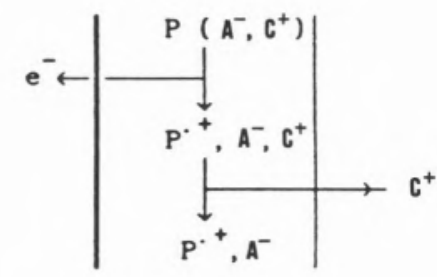

REDUÇÃO
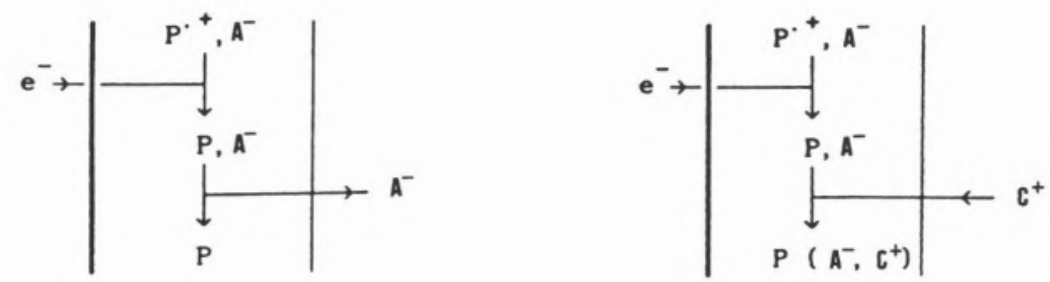

Fig. 5 - Esquema das diferentes possibilidades de inserção / expulsão de iões no processo redox de um PEC.

$\mathrm{Na}$ investigação do comportamento electroquímico de PEC, os registos simultâneos das respostas da corrente eléctrica e da deflexão do feixe laser permitem reconhecer quatro modos de transporte iónico, como se ilustra na figura $\mathbf{6}$, obtida para um filme de polianilina em so-

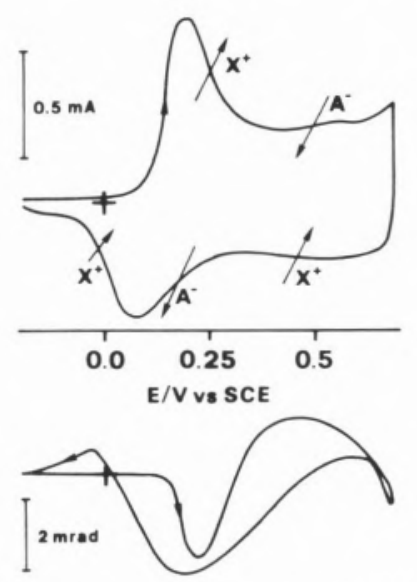

Fig. 6 - Voltamograma cíclico e deflectograma de um filme de polianilina em Pt numa solução $1.0 \mathrm{M} \mathrm{HCl}$. lução de ácido clorídrico $1 \mathrm{M}$. O deflectograma na zona do potencial correspondente à oxidação do polímero é negativo, indicando a expulsão de catiões da matriz polimérica para a solução. A valores de potencial mais anódicos a deflexão torna-se positiva manifestando que a oxidação da polianilina é acompanhada pela inserção de aniões no filme.

Durante o varrimento inverso de potencial, após o desvio positivo, dá-se a inversão do sinal do deflectograma evidenciando a ocorrência da inserção de catiões e da expulsão de aniões durante a redução do polímero.

Resultados desta natureza têm permitido discutir mecanismos propostos com base em dados puramente electroquímicos, como é o caso da primeira oxidação da polianilina aqui ilustrada [9], esclarecer a influência da natureza e composição do electrólito no comportamento redox de polímeros condutores [20,21] e confirmar a imobilização de aniões específicos em matrizes poliméricas $[22,23]$, aspecto de interesse cres- 
cente devido à possibilidade de aumentar a actividade electrocatalítica destes materiais.

\section{Estudo de uma reacção electrocatalítica}

A aplicação do Efeito Miragem ao estudo da electro-oxidação do ião hipofosfito, em meio alcalino, num eléctrodo de níquel, é ilustrativo da possibilidade de estudar o mecanismo reaccional de reacções electrocatalíticas em sistemas que envolvem a participação de duas espécies solúveis em fluxos de sentidos opostos. Embora a investigação deste sistema por técnicas puramente electroquímicas [24], tenha demonstrado a existência de uma etapa lenta, não faradaica, que antecede a oxidação do ião hipofosfito (reacção 9 e 10),

$\mathrm{H}_{2} \mathrm{PO}_{2}^{-} \rightarrow \mathrm{H}_{2} \mathrm{PO}_{2}^{-}$(ads)

$\mathrm{H}_{2} \mathrm{PO}_{2}^{-}(\mathrm{ads}) \rightarrow \dot{\mathrm{H}}($ ads $)+\mathrm{H}_{\mathrm{PO}_{2}^{-}}$(ads)

$\dot{\mathrm{H}}_{2}^{-}$(ads) $+\mathrm{H}_{2} \mathrm{O} \rightarrow \mathrm{HPO}_{3} 2^{-}+2 \mathrm{H}^{+}+\mathrm{e}(11)$

$\dot{\mathrm{H}}$ (ads) $\rightarrow \mathrm{H}^{+}+\mathrm{e}$

$2 \dot{\mathrm{H}}($ ads $) \rightarrow \mathrm{H}_{2}$

a aplicação da técnica do Efeito Miragem permitiu analisar a hipótese da adsorção do ião hipofosfito ser o passo determinante da velocidade reaccional [18].

A figura 7 apresenta o cronoamperograma e respectivo cronodeflectograma resultantes da aplicação de um impulso de potencial. A ligeira deflexão negativa nos primeiros instantes é atribuída à redução do hidróxido de níquel que se forma na superfície do eléctrodo assim que este é imerso em soluções alcalinas. A deflexão negativa e corrente anódica concomitantes são qualitativamente consistentes com o aumento do índice de refracção junto ao eléctrodo, resultante da formação do ião fosfito (reacçãol 1).

Relativamente ao desvio positivo, a hipótese deste resultar da adsorção do ião hipofosfito foi avaliada por estudos do efeito da variação da

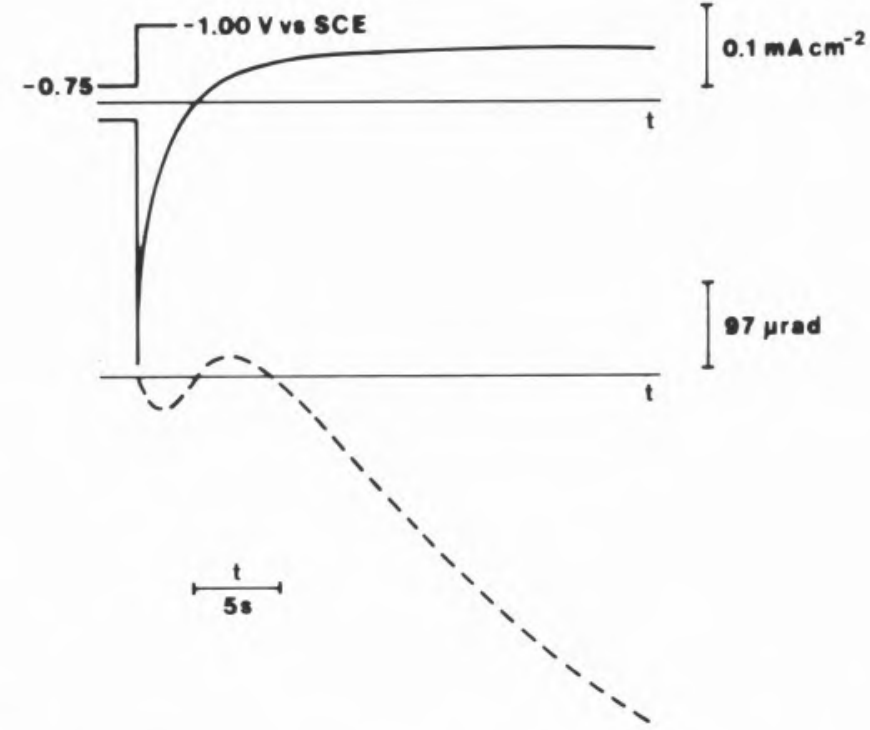

Fig. 7 - Cronoamperograma e cronodeflectograma de um eléctrodo de $\mathrm{Ni}$ em $0.05 \mathrm{M} \mathrm{NaH}_{2} \mathrm{PO}_{2}+0.1 \mathrm{M} \mathrm{NaOH}^{*}$.

concentração do agente redutor, da distância feixe-eléctrodo e da comparação da curva experimental da deflexão do feixe com a prevista por um modelo teórico.

Os resultados foram conclusivos de que os iões hipofosfito devem encontrar-se adsorvidos desde o limite positivo de potencial, mesmo sobre uma superfície oxidada, excluindo, deste modo, a hipótese da adsorção do ião hipofosfito como passo determinante da velocidade reaccional.

\section{PERSPECTIVAS FUTURAS}

Embora até ao momento a aplicação do Efeito Miragem tenha sido essencialmente limitado ao estudo mecanístico de polímeros electronicamente condutores e oxidação/redução de metais, é grande a diversidade de processos electroquímicos que poderão ser, no futuro, investigados por esta técnica. Salienta-se o estudo da bioelectroquímica, da electrosíntese de compostos orgânicos e inorgânicos, da preparação de novos materiais e da electrocatálise.

Deverá ser igualmente investigada no futuro a possibilidade de uti- lização de eléctrodos não planares, eventualmente esféricos, permitindo assim uma maior aproximação do feixe laser à superfície do eléctrodo, e consequentemente uma maior sensibilidade à monitorização de movimentos iónicos na superfície do material condutor, viabilizando assim, estudos de adsorção e de electroanálise.

$\mathrm{O}$ desenvolvimento futuro do Efeito Miragem também deverá estar, em grande medida, associado ao seu acoplamento a outras técnicas de investigação "in-situ", que possibilitam a obtenção de informação complementar sobre a estrutura e composição da interface electroquímica. São disso exemplo os trabalhos já efectuados da conjugação desta técnica à microbalança de quartzo [25] e à deflexão fototérmica [26].

Pese embora o papel relevante da técnica Efeito Miragem na obtenção, em termos qualitativos, de informação mecanística, é importante o desenvolvimento de fundamentos teóricos que permitam uma simulação das curvas de deflexão em condições experimentais cada vez mais próximas das reais, permitindo assim uma interpretação quantitativa mais rigorosa dos resultados experimen- 
tais. Neste sentido, são de registar os estudos já efectuados sobre o efeito do diâmetro de feixe laser [5], da presença do electrólito suporte [5], do contra-ião [27] e do efeito de migração [7].

\section{* CECUL, Faculdade de Ciências da Universidade de Lisboa, Rua da Escola Politécnica 58 , 1294 Lisboa Codex, Portugal TEL: (01)7573141 / FAX (01)7500115 e-mail: Luisa.Abrantes@fc.ul.pt \\ † Secção de Química, Universidade de Trás-os-Montes e Alto Douro, Apartado 202, 5001 Vila Real Codex, Portugal TEL: (059)321631 / FAX (059)320480 e-mail:mcris@marao.utad.pt}

Re-impresso do jornal Electrochimica Acta, vol.40, N 7, G. M. Brisard, I. D. Rudnicki, F. McLarnon e E. I. Cairns, Application of Probe Beam Deflection to study the electrooxidation of copper in alkaline media, pp. 859-865, Copyright (1995), with kind permission from Elsevier Science Ltd, The Boulevard, Langford Lane, Kidlington 0X5 1GB, UK.

\section{REFERÊNCIAS}

1. A. C. Boccara, F. Fournier e I. Badoz, Appl. Phys, Lett., 36 (1980) 130

2. G. H. Brilmver e A. I. Bard, Anal. Chem., 52 (1980) 685

3. I. Pawliszyn, M. Weber, M. Diagnam, A. Mandelis, R. Venter, S. M. Park, Anal. Chem., 58 (1985) 236.

4. R. Koetz, C Barbero e O. Haas, Ber. Bunsenges. Phys. Chem. 97 (1993) 427.

5. I. Rudnicki, G. M. Brisard, H. A. Gasteir, R. Russo, F. R. McLamon e E. J. Cairns, I. Electroanal. Chem., 362 (1993) 55.

6. M. F. Mathias, J. Electroanal. Chem., 407 (1996) 115

7. V. Plinchon, R. Even e G. Beiner, 1. Electroanal, Chem. 305 (1991) 195

8. E. Vieil, K. Meerholz, T. Matencio e I. Heinze, I. Electroanal. Chem., 368 (1994) 183.

9. C. Barbero, M. C. Miras, O. Haas e R. Koetz, I. Flectrochem. Soc. $138(1991) 669$.

10. C. Lopez, M. F. Mendes Viegas, G. Bidan e E. Vieil Synth. Met., 63 (1994) 73.

11. C. Barbero, M. C. Miras, R. Koetz and O. Haas, Solid State lonics, $60(1993) 167$

12. C. Liu, G. Cheng, I. Li, I. Jin, S. Dong, J. Electroanal. Chem., 407 (1996) 243.

13. F. Decker, R. Neuenschwander, C Cesar e A. Penna, L. Electroanal Chem, 228 (1987) 481.

14. R. Russo, F. McLarnon, J. Spear e E. Cairns, J. Electrochem. Soc, 134 (1987) 2738
15. L. M. Rosolen, F, Decker, M. Fracastoro-Decker, R. M. Torresi e S. I. Cordoba de Torresi, , I. Electroanal. Chem, 354 (1993) 273

16. P. Sébastien, DEA na Université de Paris VI, 1991.

17. V. Kertész, G. Inzelt, C. Barbero, R. Koetz e O. Haas, I. Electroanal Chem, 392 (1995) 91.

18.L. M. Abrantes, M. C. Oliveira e E. Vieil, Electrochimica Acta, 41 (1996) 1515

19. G. M. Brisard, 1. D. Rudnicki, F. McLarnon e E. I. Cairns, 1. Flectrochem. Soc 40 (1995) 859

20. V. W. Iones, Tese de Doutoramento, Universidade de Wales (1995)

21. L. M. Abrantes, I. P. Correia e I. Gonzalez comun ás IPC'97: t Chimie Physique et de Physico-Chimie Biologique, em publicação.

22. V. W. Jones, M. Kalaji, G. Walker, C. Barbero e R. Koltz, 1. Chem. Soc. Faraday Trans, 90 (1994) 2061.

23. L. M. Abrantes, V. W. lones, M. Kalaii, I. Chem. Soc, Fdraday. Trans, submetido.

24. L. M. Abrantes e M. C. Oliveira, Electrochimica Acta, 41 (1996) 1703

25. Christian Lopez, Tese de Doutoramento na Universite loseph Fourier-Grenoble 1, 1994.

26. R. Koetz, C. Barbero e O. Haas, 1. Electroanal. Chem., 296 (1990) 37

27. M. A. Vorotyntsev, C. Lopez e E. Vieil, I. Electroanal. Chem, 368 (1994) 155.

\section{Instituto Português da $\mathbf{Q}$ ualidade}

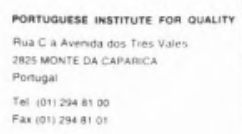

CERTIFICADO

DE CONFORMIDADE

\section{6/CEP.410}

O INSTITUTO PORTUGUÊS DA QUALIDADE certifica que o Sistema da Qualidade da

\section{SOQUIMICA - SOCIEDADE DE REPRESENTAÇÕES DE}

\section{QUIMICA, LDA.}

Rua Coronel Santos Pedroso, 15

1500 LISBOA

PORTUGAL

implantado na comercialização, manutenção e calibração de equipamento de laboratório, cumpre os requisitos da Norma Portuguesa NP EN ISO 9002 - "Sistemas da Qualidade. Modelo de garantia da qualidade na produção, instalação e assistência após venda".

O presente certificado é emitido ao abrigo do Decreto-Lei n. ${ }^{\circ} 234 / 93$ de 2 de Julho, e de acordo com a Directiva CNQ 22 - "Certificação de sistemas da qualidade de empresas. Metodologias e regras gerais".

A presente certificação é válida por um período de três anos, renovável.

Monte de Caparica, 18 de Junho de 1996

\section{Caindid on Sauto}

